\title{
Turismo sustentable:
} Investigación en la Universidad de Quintana Roo, Campus Cozumel

\author{
DOI: 10.22403/UQROOMX/TYP01/07
}

Resumen

Alejandro Palafox Muñoz

El desarrollo sustentable ha sido, en últimas fechas, un tópico relevante que indiscutiblemente se vincula con toda actividad que tenga por insumo básico los recursos naturales y culturales. El programa de investigación Desarrollo Turístico Sustentable de la Universidad de Quintana Roo (Uqroo), Campus Cozumel, pretende abordar una gran gama de objetos de estudio con la finalidad de generar información y marcar las pautas para posteriores investigaciones, para tal efecto se propone al diagnóstico como aquella herramienta que proporciona los datos necesarios como insumo de futuras indagaciones, contribuyendo de esta forma a la misión institucional de la Uqroo para consolidarse como la punta de estudios sobre este tema en el Caribe.

Palabras $\mid$ Universidad de Quintana Roo, Cuerpo Académico de Turismo,

CLAVE investigación turística, turismo sustentable, desarrollo turístico sustentable.

*Universidad de Quintana Roo / palafox@correo.uqroo.mx 
Turismo sustentable:

Investigación en la Universidad

de Quintana Roo

\section{Introducción}

Sistema, fenómeno e industria, en el turismo, son términos a tomar en cuenta en la investigación de un objeto de estudio sobre el tema; entrañan un análisis de los impactos que genera la actividad turística, sus múltiples incidencias en el entorno, no solamente en el aspecto económico, sino político, en el contexto social, cultural, las repercusiones ambientales; la percepción de la comunidad receptora, del visitante, las autoridades y de la iniciativa privada; de igual forma su papel en la economía internacional, nacional, regional y municipal, sin olvidar el enfoque administrativo y mercadológico.

Los tópicos anteriores tendrán que ser descritos, analizados e interpretados previo a la elaboración de un programa que marque las pautas para el desarrollo sustentable de la actividad que, para tal efecto, tendrá que ser congruente con el entorno natural, social y económico, y los esfuerzos que se lleven a cabo comprendan el ámbito local, regional y nacional.

De la discusión y el análisis nace este programa de investigación que tiene como finalidad proponer las pautas para el desarrollo turístico sustentable, cuyo propósito es responder al desequilibrio que por las tendencias del turismo presentan las localidades, regiones, el país y el mundo en conjunto; lo anterior con el objetivo de "satisfacer las necesidades de la generación presente sin comprometer la capacidad de las generaciones futuras para satisfacer sus propias necesidades" (Comisión Mundial del Medio Ambiente y del Desarrollo, 1992:67).

De esta manera y con la intención de identificar la situación que se manifiesta en la actividad turística, es necesario elaborar los diagnósticos que aporten elementos para la toma de decisiones que puedan ser traducidas en acciones que corrijan el rumbo del sector; esto es, generar los insumos básicos para el impulso de la sustentabilidad de una actividad que por ahora se desarrolla en forma desordenada y ocasiona el incremento de la desigualdad en quienes dependen del turismo con consecuencias estructurales que pueden ser irreversibles.

Este programa concentra una serie de temáticas que serán abordadas mediante diagnósticos elaborados con un instrumento que sirve para la investigación y evaluación del turismo, formulado por la Universidad George Washington y el organismo Conservación Internacional, el cual "está diseñado 
por un equipo multidisciplinario, en conjunto con los agentes locales, con el propósito de presentar un análisis del destino turístico, al mismo tiempo de ser utilizado para la planeación del desarrollo turístico" (George Washington University: I). Tourism Rapid Assessment Tool es un instrumento útil para el análisis e interpretación de la actividad turística, ya que permite la integración de un equipo multidisciplinario de profesionales para el estudio del turismo y conduce la identificación de las incidencias que tiene el fenómeno en la actividad económica, social y ambiental.

En este sentido los estudios se enfocarán en los parámetros de planificación turística sustentable (ОМт: 1999) de carácter global, que reúna los componentes característicos del sistema (oferta y demanda), el cual integre las políticas y planes de desarrollo local, estatal y federal con el propósito de concentrarse en la participación activa de las comunidades. Para que el enfoque esté dirigido a las personas es necesaria la identificación de los problemas que atañen al turismo y proponer las directrices que marquen su progreso.

\section{El Programa de Investigación}

En la cumbre mundial de Johannesburgo,Vicente Fox Quesada afirmó que el país está comprometido con el desarrollo sustentable y sostenible, por lo que ya no se puede permitir el crecimiento económico a costa del abuso de los recursos del planeta o de la exclusión social.

Alcanzar el desarrollo sustentable es una misión compartida donde la suma de voluntades y la cooperación de los diferentes actores: sector público, privado y sociedad civil, son imprescindibles para asegurar la supervivencia de la humanidad. Tanto en la Carta de la Tierra como en la Agenda 2 I para el turismo mexicano, se confirma la importancia de impulsar al Turismo como una actividad económica que sea sustentable y responsable, como condición para lograr el desarrollo a largo plazo [SECTUR, SEMARNAT, 2002:3].

Asimismo, la Agenda 2 I, se convierte en el documento donde convergen estrategias y acciones a corto, mediano y largo plazo, con el propósito de fortalecer las regiones turísticas, mediante la inclusión de las comunidades receptoras en las actividades económicas que se generen, a fin de que los recursos naturales y culturales sean preservados. 
Turismo sustentable:

Investigación en la Universidad

de Quintana Roo

En este sentido, "el municipio tiene un papel protagónico, ya que funciona como promotor estratégico para atender los desafíos ambientales locales, también como el gran conductor en los procesos de cambio y enlace entre los grupos que interactúan en los procesos locales de desarrollo económico, social y cultural" (Ibid: 4), dejando así el papel de espectador y transformándose en fuente activa de propuestas para la toma de decisiones, además de fomentar los catalizadores que promuevan el desarrollo de las comunidades.

Por tales motivos, el programa de investigación está dirigido a promover los diagnósticos situacionales que contribuyan al fomento del desarrollo turístico sustentable y coadyuven a la obtención de información básica para la planeación de la actividad, de ahí que los trabajos estarán enfocados a identificar los atractivos naturales y culturales, analizar el entorno económicopolítico que se suscita alrededor de la actividad turística, conocer la infraestructura con que cuenta la localidad misma que es utilizada con fines turísticos y de servicio a la población; identificar el mercado turístico en el que compite el destino; así como la percepción que tiene el turista del atractivo; analizar los impactos que tiene el turismo en la localidad receptora; conocer la incidencia ambiental que se presenta en los recursos naturales y culturales del destino turístico, entre otros.

De esta forma, planear la actividad turística tiene por objeto que ciertos beneficios de índole económico y social sean vertidos a la sociedad y consecuentemente auxilie al desarrollo sustentable del sector turístico a través de la protección ambiental y de la cultura local. Sin embargo, algunas comunidades cuentan con recursos limitados para el desarrollo del turismo, lo que no impide su impulso, sino que exige comprender el impacto que éste tendrá en la localidad, para el futuro de la misma.

Por ello, en la planeación el punto de partida es la elaboración de un diagnóstico, por lo que los objetivos e intervención que se persigan estarán en función de los alcances y contenido del mismo, de ahí su complejidad para la toma de decisiones, por lo que éste debe contener:

- Una caracterización de la naturaleza de la magnitud de los problemas, sin perder de vista su inserción en el contexto global en que aparecen las relaciones y determinaciones que dan origen y explican la existencia de tales problemas. 
- Es necesario incluir una evaluación de las acciones realizadas o en proceso que se vinculen con el problema que se trate.

- Un examen de la actuación de las fuerzas y grupos sociales en la escena política [Pichardo, 1994:97].

Para llevar a cabo lo anterior es necesario incluir información documental, indicadores, inventarios, etc., los cuales proporcionan una aproximación de los problemas para centrarse en "identificar las posibles determinaciones y relaciones causales que inciden en la producción de fenómenos sociales" (ibid: 98), tal es el caso del turismo, lo que lleva a delimitar sus acciones.

Por tanto, para que la investigación del turismo pueda integrar y propiciar beneficios a todos los sectores de la población, es necesario que ésta sea vista con un enfoque global, integral y basado en la comunidad, es decir que:

todos los aspectos del desarrollo turístico deben tomarse en cuenta; además de las políticas y planes generales de desarrollo local, de igual forma se integran las políticas y planes regionales y nacionales de turismo; centrándose en la identificación y resolución de cuestiones inmediatas; y en la medida posible procurarse la máxima participación de las comunidades locales, de forma que los beneficios recaigan en las mismas comunidades, incluso los grupos menos afortunados y las minorías [OMT, 1999: 46-47].

De esta manera, y con el propósito de lograr que el turismo se convierta en una actividad sustentable, el programa de investigación pretende contar en primera instancia con los insumos necesarios producidos de la elaboración de diagnósticos situacionales; para tal efecto éste divide las áreas de estudio en cuatro grandes apartados:

\section{Turismo, planeación y desarrollo}

Entre las temáticas que comprende este apartado figuran aquellas referidas a algunos países que han encontrado en la actividad turística una forma de vida y sustento económico, por lo que para su progreso es necesario planearlo, crear políticas, promoverlo, diagnosticarlo, analizarlo e interpretarlo. El turismo ha sido impulsado y manejado por dos actores sociales, hoy en día por los empresarios y anteriormente por el gobierno federal, sin embargo, los roles han cambiado, por lo que la formulación de políticas, planes, programas y proyectos toman un nuevo curso, mismo que es de interés analizarlo por su complejidad y escasa información. 
Turismo sustentable:

Investigación en la Universidad

de Quintana Roo

Asimismo se podrán investigar las relaciones existentes entre el sector público y el turismo, su incidencia, desarrollo y perspectivas.

También se pretende indagar sobre la existencia de legislaciones, reglamentos, normas, planes, programas y proyectos que contemplen o incidan en el desarrollo de la actividad turística sustentable; definir cual ha sido la política turística que ha seguido el municipio con respecto al turismo en la comunidad, así como los aspectos relacionados con el poder local y su incidencia en el turismo, es decir, quienes toman las decisiones sobre el rumbo al cual va dirigida la actividad.

La localidad necesita de información económica y demográfica tanto de la comunidad receptora como del visitante, ejemplo de los datos que han de reunirse son el número de visitantes, edades, ingresos, profesión, motivo del viaje, lugar de residencia, tipo de transporte utilizado, estadía promedio, gasto promedio, impacto económico del turismo, servicios complementarios, número de hoteles, restaurantes, arrendadoras, agencias de viajes, etc., además de desarrollar la información referente a los aspectos geográficos, demográficos, clima, perfil poblacional, desarrollo económico, religión, sistema político, mapas, etcétera.

\section{Mercadotecnia e industria turística}

En últimas fechas, la mercadotecnia ha sido la herramienta más utilizada para la elaboración de investigaciones relacionadas con el turista, las ventas, distribución, productos, promoción, entre otras, a través de la búsqueda de causas, descripción y creación de nuevos consumidores. De esta forma, el negocio del turismo ha sido investigado y sus resultados difundidos mediante reportes que reflejan estadísticamente su funcionamiento, en ocasiones en comparación con otros destinos o bien con empresas dedicadas al ramo. Estos productos de investigación son de carácter descriptivo, con la finalidad de aportar elementos que coadyuven en la toma de decisiones; dentro de las temáticas a abordar se encuentran los estudios de demanda, desarrollo de soluciones a problemas dados, intervención del gobierno en el impulso a la industria turística, procedimientos, entre otros; de ahí la necesidad del mercado potencial y cautivo del lugar, basado en el perfil sociodemográfico del visitante, analizando el interés y satisfacción sobre el destino, identificando los mejores mercados para el futuro desarrollo, así como obtener el mercado por estado y país de origen, segmentación turística, entre otros aspectos. 
Por otra parte, dependiendo del destino en estudio, se realizarán los inventarios de los recursos naturales y culturales, con base en el tipo de mercado que se pretende alcanzar, así como aquellas atracciones que son producidas por el hombre: eventos, actividades recreativas, deportivas, etcétera, además lo relacionado con la accesibilidad al destino, comunicaciones, tratamiento de aguas y residuos.

En lo referente a la red de transportes (aeropuerto, barco, terminales de autobuses, señalización, carreteras, servicios de taxis, estacionamientos, transporte urbano, tipo de transporte utilizado por el visitante, corridas regulares y su enlace con otros medios, horarios fijos, etc.), y con respecto a las comunicaciones, se necesita saber lo concerniente al servicio telefónico nacional, internacional, Internet, e-mail, telefonía móvil, entre otros, así como el acceso al servicio de agua potable, alcantarillado, seguridad, disponibilidad, tratamiento, eliminación de residuos, eliminación y reciclaje de sólidos.

Del mismo modo, conocer el número de establecimientos de hospedaje, alimentación, agencias, arrendadoras, oferta alternativa, souveniers, cantidad de cuartos, de negocios, tipo de servicio, categoría, renta diaria, promedio de ocupación, tipo de visitante, estancia promedio, propósito de la visita, propietario, administración, número de empleados y promoción; con el objetivo de observar la diversificación de la oferta y venta de servicios.

Además, habrá que identificar si la comunidad receptora cuenta con los servicios y preparación para responder a las demandas de los visitantes, lo que define la calidad del servicio que se vende, así como la necesidad de los empleadores por capacitarse; se obtiene información de los estudios relacionados con la satisfacción del turista, impresión del destino, evaluaciones de los administradores, programas existentes de entrenamiento y quién proporciona dicha capacitación, asimismo si la localidad cuenta con escuelas que provean ese servicio o se acude a instancias foráneas.

También se puede llevar a cabo la revisión de las organizaciones turísticas y su estructura jerárquica, las responsabilidades que tienen las oficinas de turismo y los niveles de interacción, presupuesto para el financiamiento turístico sustentable, su coordinación con las entidades federales, asociaciones profesionales del área en la localidad o en la región, ONG's articuladas con el impulso de la actividad, acuerdos sostenidos por organismos dedicados a la promoción turística en colaboración con la comunidad; asimismo, describir 
Turismo sustentable:

Investigación en la Universidad

de Quintana Roo

cuál es la relación existente entre ellas, los proyectos que se plantean para el destino en estudio, así como su perspectiva de inversión, destacando los principales competidores en la región, productos y servicios ofertados en el destino, ventajas competitivas, accesibilidad, precio, empresas multinacionales establecidas en la competencia, incentivos proporcionados por el gobierno local y organización dedicada al mercadeo de productos.

\section{Turismo y medio ambiente}

El turismo y el medio ambiente están íntimamente relacionados, el entorno natural $\circ$ el construido por el hombre, ofrece atractivos que la actividad turística utiliza. Por su parte, el desarrollo turístico genera impactos positivos y negativos sobre el medio ambiente. El desarrollo sustentable del turismo depende del uso adecuado de los recursos naturales y de la participación en la preservación, conservación y cuidado de la identidad local en relación con éstos. Los puntos específicos de las investigaciones en medio ambiente y sustentabilidad en el turismo serán abordados desde la perspectiva geográfica (territorio, tiempo libre, evaluación de los recursos naturales físicos, sociales y económicos) y ecológica (evaluación de los recursos naturales renovables, ecología, recreación en áreas naturales y su impacto), centrándose en el medio natural y su relación con los aspectos social y cultural, para el desarrollo local y en el marco de la política ambiental.

\section{Turismo y sociedad}

El turismo ha sido estudiado por distintas disciplinas, principalmente por la sociología, la antropología, la geografía e historia, lo que ha generado una gran variedad de investigaciones cuyo objeto de estudio es el hombre con el entorno turístico y sus incidencias.

En este apartado se comparte y analiza el conocimiento turístico sobre el destino y los puntos de vista que tiene la población con respecto a la actividad y el desarrollo que ésta provee a la comunidad, la existencia de fomento al desarrollo turístico sustentable, la división entre los promotores del turismo y la localidad, la preocupación de los residentes por el rumbo que toma la política turística del lugar, la existencia de beneficio directo a la comunidad receptora, la vida cotidiana, la práctica turística, los cambios en la cultura, la pérdida de tradiciones, entre otros aspectos. 
El desarrollo turístico sustentable puede tener impactos positivos y negativos en la sociedad, en la economía, en la naturaleza y en el ambiente local. En el diagnóstico económico se necesita conocer al menos las estimaciones con respecto al total de visitantes por día, gasto, monto en operación y desarrollo de proyectos que provean el mantenimiento de servicios públicos de fluctuación de la fuerza de trabajo en temporadas. Con respecto a lo sociocultural, se revisa el incremento o baja de la población, migración, fuente y niveles de ingreso, estructura poblacional, estructura laboral, estilo de vida local, oportunidades recreacionales y de entretenimiento para la sociedad.

\section{Conclusión}

La Unidad Cozumel de la Universidad de Quintana Roo ha formulado dos proyectos de investigación, uno en la temática de turismo y medioambiente, y el segundo en su relación con la planeación y el desarrollo, fortaleciendo así la misión de la Unidad al contribuir con el desarrollo social, económico, cultural y profesional de la entidad, sin embargo, es necesario atacar las temáticas relacionadas con la empresa y la población, aspectos que por el momento no han sido abordados.

La amplitud de aspectos que pueden investigarse en este programa es vasta, solamente hace falta el interés de los profesores investigadores para que se vinculen a esta propuesta y comience el proceso de consolidación del Cuerpo Académico de Turismo, en lugar de realizar esfuerzos aislados -producto del hedonismo académico-, por lo que es necesario recordarles que el turismo es una actividad de índole social, económica y ambiental, en donde el turista, la población local, los prestadores de servicios y el municipio son entes que se interrelacionan y cada uno de ellos cuenta con una estructura diferente, es decir, que las aproximaciones pueden ser variadas e infinitas.

El interés personal y el de la institución son similares, es decir, el turismo, tópico que puede ser abordado desde múltiples perspectivas, entre las que destacan la social, antropológica, económica, administrativa, mercadológica, ambiental, ecológica y geográfica, entre otras. Esto hace posible que el fenómeno, industria o sistema proporcione varias y amplias opciones para su 
Turismo sustentable:

Investigación en la Universidad

de Quintana Roo

aproximación, lo que invita a realizar trabajos multidisciplinarios e interinstitucionales, con el objetivo de progresar en la construcción del conocimiento relacionado con el desarrollo turístico sustentable.

\section{Fuentes consultadas}

Ander-Egg, Ezequiel (1998). Metodología y práctica del desarrollo de la comunidad I. Buenos Aires: Hvmanitas.

CESTA (2002). El concepto de sustentabilidad. Octubre, www.cesta-foe.org

Comisión Mundial del Medio Ambiente y del Desarrollo (1992). Nuestro futuro común. España: Alianza Editorial.

Escalante Fortón, R., y Miñano G., Max (2000). Investigación, organización y desarrollo de la comunidad, México: Colofón.

George Washington University. Tourism rapid assessment tool: concept paper. Washington: GWU.

Guimaraes, Roberto (1994)."El desarrollo sustentable: ipropuesta alternativa o retórica neoliberal”. Eure, vol. XX, núm. 6I.

Jiménez Martínez, Alfonso de Jesús (1998). Desarrollo turístico y sustentabilidad, México: Porrúa.

Leff, Enrique (1996). Economía y democracia: alternativas para el desarrollo sustentable y equitativo. México: Sustentabilidad y Desarrollo Ambiental, SemARNAP.

Masri de Achar, Sofía (1997). La industria turística hacia la sustentabilidad. México: Diana.

OMT (1999a). Agenda para planificadores locales: turismo sostenible y gestión municipal. Madrid: Organización Mundial del Turismo.

----- (1999b). Guía para administraciones locales: desarrollo turístico sostenible. Madrid: Organización Mundial del Turismo.

Pichardo Muñiz, Arlette (1994). Planificación y programación social. Costa Rica: Hvmanitas, Universidad Nacional de Costa Rica.

Poder Ejecutivo Federal (200I). Programa Nacional de Turismo 200I-2006. México: Poder Ejecutivo Federal.

Provencio Durazo, Enrique (1997). “Desarrollo sustentable de las ciudades”. Ciudades, núm. 34, Puebla, abril-junio.

Secretaría de Turismo (2002a). El turismo en México 200I. México: SECTUR. 
(2002b). Resultados de la actividad turística del año 200I. México: SECTUR.

y Centro de Estudios Superiores en Turismo (200I). Estudio estratégico de viabilidad del segmento de ecoturismo en México. México: SECTUR-CESTUR.

------------------- y Secretaria del Medio Ambiente y Recursos Naturales (2002). Agenda 2 I para el turismo mexicano. México: SECTURSEMARNAT.

Serrano Barquín, Rocío del Carmen (1997). "El papel de la mujer en el desarrollo sustentable”. Ideas, núm. I3, Toluca.

Serrano Barquín, Rocío del Carmen. "Fundamentos para la planeación del turismo sustentable, hacia el desarrollo local". Periplo Sustentable, núm. 2, www.uaemex.mx

Urquidi, Víctor (1996). México en la globalización: condiciones y requisitos de un desarrollo sustentable y equitativo. México: Fondo de Cultura Económica. 